\title{
Effects of dopaminergic drug adjustment on executive function in different clinical stages of Parkinson's disease
}

This article was published in the following Dove Press journal:

Neuropsychiatric Disease and Treatment

25 October 2017

Number of times this article has been viewed

\section{Hidetomo Murakami \\ Tetsuhito Nohara \\ Hidenobu Shozawa \\ Yoshiyuki Owan \\ Takeshi Kuroda \\ Satoshi Yano \\ Machiko Kezuka \\ Mitsuru Kawamura \\ Kenjiro Ono}

Department of Neurology, School of Medicine, Showa University,

Tokyo, Japan
Correspondence: Kenjiro Ono Department of Neurology, School of Medicine, Showa University, I-5-8 Hatanodai Shinagawa-ku, Tokyo 142-8666, Japan

Tel/fax +8I 337848710

Email onoken@med.showa-u.ac.jp
Background: Effects of dopaminergic medication on executive function in patients with Parkinson's disease (PD) are inconsistent.

Objective: We examined the effect of dopaminergic medication on executive function in 24 drug-naïve PD patients (de novo group) and in 21 PD patients on chronic dopaminergic medication (chronic medication group).

Methods: PD patients without dementia were included in this study. For the de novo group patients, dopaminergic medication was initiated, and the dose was increased to improve motor symptoms. For the chronic medication group patients, dopaminergic medication was adjusted to relieve clinical problems. All participants were tested prior to and at 4-7 months after the drug initiation/adjustment. Executive function was assessed by using the Behavioral Assessment of the Dysexecutive Syndrome (BADS). Motor function was assessed by using the Unified Parkinson's Disease Rating Scale (UPDRS; part III). Improvement in executive function was compared with a simultaneous change in levodopa equivalent doses (LED) of dopaminergic medication and with improvement in motor functions.

Results: The mean standardized BADS scores showed no significant improvement in both the groups. In the de novo group, percent improvement in the standardized BADS scores showed a significant positive correlation with the LED, but not with percent improvement in UPDRS part III. In the chronic medication group, percent improvement in the standardized BADS scores was negatively correlated with change in the LED, but not with percent improvement in UPDRS part III. Multiple regression analysis using improvement in the standardized BADS score as a dependent variable and patient's background factors (ie, age, education, disease duration, and motor and executive assessments at baseline) as independent variable showed that improvement in the executive assessment is significantly correlated with the LED only in the de novo group.

Conclusion: Effects of dopaminergic drug adjustment on executive function differ according to the patient's clinical stage and depend on LED in de novo stage.

Keywords: motor disorders, dopaminergic drug, levodopa equivalent dose, correlation between executive improvement and levodopa equivalent dose, inverted U-shaped theory

\section{Introduction}

Since the 1960s, dopaminergic drugs have been used to treat motor symptoms in patients with Parkinson's disease (PD). In recent years, cognitive impairment in PD has attracted interest, and the majority of PD patients show mild cognitive impairment. Among the cognitive changes, executive deficits are frequent in PD patients. ${ }^{1,2}$ Frontal lobe functions, such as executive function, are shown to correlate with striatal uptake of ${ }^{123}$ I-Ioflupane in the dopamine transporter scintigram. ${ }^{3,4}$ Therefore, dopaminergic 
drugs have a possibility to affect not only motor function, but also executive function. Although dopamine replacement therapy is the gold standard for motor symptoms, its effects on cognitive function in PD patients are inconsistent and not well defined. For example, levodopa is shown to improve, diminish, or not affect cognitive function according to the neuropsychological tests and domains. ${ }^{5}$ For frontal lobe function, some reports show working memory, ${ }^{6}$ planning, and set-shifting ${ }^{7}$ improved in patients taking levodopa. Cools and D'Esposito showed that a dopaminergic drug causes beneficial or detrimental effects on frontal/executive function according to basal dopamine levels in the brain, because the cognitive performance becomes the best at the optimum dopaminergic titer in brain and declines at either too little or too much dopaminergic titers. ${ }^{8}$ This theory is named the "inverted U-shaped theory" and means an increase or a decrease in dopaminergic titer results in reduced executive function in patients with appropriate baseline dopaminergic titers. However, a depleted baseline dopaminergic titer supplemented with appropriate prescribed drugs results in appropriate dopaminergic titers and improved executive function. This theory is supported by later performed human studies showing association between frontal/executive function and dopaminergic titer in brain..$^{9,10}$ Thus, appropriate adjustment of dopaminergic drugs is recommended. These findings suggest that the dopamine titer has a relationship with executive function in PD patients. However, the details on the relationship between executive function and dopamine titer in clinical practice are not known because dopaminergic drugs are primarily used to treat motor function in clinical practice.

Previous studies examined executive function in PD using standard assessments, such as the Stroop test, the Wisconsin card sorting test, the Frontal Assessment Battery, and others. ${ }^{11-14}$ However, these assessments may be insensitive to executive deficits experienced in daily life. ${ }^{15}$ However, the Behavioral Assessment of the Dysexecutive Syndrome (BADS), which includes six subtests to assess executive function required in daily life (Table 1), is designed to examine subtle impairment in executive function. ${ }^{16}$ Therefore, it is useful to identify executive deficits in patients with no apparent dementia such as Mini-Mental State Examination (MMSE) score of $\geq 24 .{ }^{17,18}$ Usefulness of the BADS to assess executive function in PD has been shown. ${ }^{18-20}$ Using BADS, we examined the effects of dopaminergic medication on executive function in nondemented PD patients at two different clinical stages, including the de novo stage and the more progressed stage with a history of chronic dopaminergic medication.

\section{Methods}

\section{Patients and treatment}

In total, $45 \mathrm{PD}$ patients with an MMSE score of $\geq 25$ were recruited from both outpatients and inpatients diagnosed at both Showa University Hospital and Showa University East Hospital in Tokyo, Japan. The patients were divided into two groups. The de novo group included 24 de novo patients with no dopaminergic medication, while the chronic medication group included 21 patients receiving chronic dopaminergic medication and requiring further drug adjustment to manage newly developing clinical problems, such as hallucinations and motor symptoms. The cutoff MMSE score was determined because the sensitivity and usefulness of BADS are observed in patients with an MMSE score $>24 .{ }^{17,18}$ The diagnosis of PD was made using the clinical diagnostic criteria of the United Kingdom Parkinson's Disease Society Brain Bank. ${ }^{21}$ All de novo patients were diagnosed according to both their clinical history and neurological findings before medication. Then, single use or combination of dopaminergic drugs were introduced and increased stepwise to an optimal dose for improvement in motor function within

Table I Profile of six subtests included in the BADS

\begin{tabular}{|c|c|c|}
\hline Subtest & Contents of each subtest & Measurable abilites ${ }^{15,16}$ \\
\hline Rule shift cards test & $\begin{array}{l}\text { Respond "yes" or "no" according to the prescribed rules when a series of } 20 \text { playing } \\
\text { cards is turned over one at a time }\end{array}$ & Cognitive flexibility \\
\hline Action program test & Take the cork from the tall tube using a set of materials according to the prescribed rules & Planning and problem-solving \\
\hline Key search test & $\begin{array}{l}\text { Draw the path the patient would take to search inside the square in order to find a } \\
\text { lost key }\end{array}$ & $\begin{array}{l}\text { Planning and monitoring } \\
\text { patient's own behavior }\end{array}$ \\
\hline Temporal judgment test & Estimate the time length for activities experienced in daily life & Judgment and estimation \\
\hline Zoo map test & Visit the ordered places in the map according to the prescribed rules & Planning and problem-solving \\
\hline $\begin{array}{l}\text { Modified six elements } \\
\text { test }\end{array}$ & $\begin{array}{l}\text { There are two sets of arithmetic problems, two dictation tasks, and two sets of pictures } \\
\text { that have to be named, making six subtasks in total. The subject is required to attempt } \\
\text { to do at least some of all six sections within } 10 \text { minutes according to the rules. However, } \\
\text { they are not allowed to do the two parts of the same task consecutively }\end{array}$ & $\begin{array}{l}\text { Planning, problem-solving, } \\
\text { prospective memory, and } \\
\text { organizing and monitoring } \\
\text { patient's own behavior }\end{array}$ \\
\hline
\end{tabular}

Abbreviation: BADS, Behavioral Assessment of the Dysexecutive Syndrome. 
the following 3 months. The motor symptoms of all de novo patients improved, confirming their diagnosis of PD. We used the sum of the levodopa equivalent doses (LEDs) of the prescribed drugs at follow-up assessment as the change in the LED. Protocol for calculating the LED ( $\mathrm{mg} /$ day) for each drugs is as follows: pramipexole, X100; ropinirole, X20; rotigotine, X30; selegiline, X10; entacapone, levodopa dose $\mathrm{X} 0.33$; amantadine, $\mathrm{X} 1 .^{22}$ All of the patients in the chronic medication group were taking levodopa/dopa decarboxylase inhibitor (DDCI) and/or other dopaminergic drugs (ie, dopamine agonists, selegiline, entacapone, and amantadine) as shown in Table 2. For those chronic medication patients, the dopaminergic medication was adjusted, including either dose or drug change, to relieve their clinical problems, such as hallucinations and motor symptoms. The adjustment was performed stepwise within 3 months of entering the study. We calculated the total LED of the taken dopaminergic drugs at baseline and follow-up period. The change from baseline in the LED, which signifies an increase in a positive number and a decrease in a negative number, was used as the change in the LED for our analysis.

In both the groups, no patient was taking an anti-dementia drug, such as either an acetylcholinesterase inhibitor or an N-methyl-D-aspartic acid receptor antagonist. No other drug initiation/change except dopaminergic drugs was performed

Table 2 Patients' backgrounds in both the groups

\begin{tabular}{|c|c|c|}
\hline & $\begin{array}{l}\text { De novo } \\
\text { group }\end{array}$ & $\begin{array}{l}\text { Chronic } \\
\text { medication group }\end{array}$ \\
\hline \multicolumn{3}{|l|}{ Sex } \\
\hline Male/female & $11: 13$ & $8: 13$ \\
\hline Age (years) & $65.8 \pm 11.2$ & $68.8 \pm 10.2$ \\
\hline Education (years) & $13.4 \pm 2.3$ & $13.0 \pm 2.0$ \\
\hline Disease duration (years) & $1.3 \pm 0.9$ & $4.6 \pm 3.8 * * *$ \\
\hline \multicolumn{3}{|l|}{ Onset symptom } \\
\hline Tremor & 10 & 14 \\
\hline Rigidity and bradykinesia & 14 & 7 \\
\hline \multicolumn{3}{|l|}{ Hoehn and Yahr } \\
\hline I & 6 & 0 \\
\hline II & 10 & 14 \\
\hline III & 8 & 7 \\
\hline IV & 0 & 0 \\
\hline V & 0 & 0 \\
\hline \multicolumn{3}{|c|}{ Number of patients taking each drug } \\
\hline Levodopa/DDCI & 0 & 16 \\
\hline Entacapone & 0 & 2 \\
\hline Selegiline & 0 & 8 \\
\hline Pramipexole & 0 & 8 \\
\hline Ropinirole & 0 & 3 \\
\hline Rotigotine & 0 & 3 \\
\hline Amantadine & 0 & $\mathrm{I}$ \\
\hline
\end{tabular}

Note: $* * * p<0.001$ vs de novo group.

Abbreviation: DDCl, dopa decarboxylase inhibitor. during this study. No patient was taking an anti-cholinergic drug, such as trihexyphenidyl. No patient had a history of an impulse control disorder. No patient in the chronic medication group was suffering from the wearing-off phenomenon. No patient had undergone deep brain stimulation. No patient had a disease, such as depression, that affected motor and cognitive functions except PD.

The Ethics Committee of Showa University School of Medicine approved the study, and the study was performed according to the Declaration of Helsinki. Written informed consent was obtained from all the participants.

\section{Clinical assessment}

The motor and executive functions of each participant were assessed at baseline and at the follow-up period of 4-7 months after drug initiation or adjustment. Motor function was assessed using the Unified Parkinson's Disease Rating Scale (UPDRS; part III). MMSE was performed to determine the cognitive level in patients with PD. Motor and neuropsychological assessments were performed blind by different examiners. For patients with an MMSE score of $\geq 25$, executive function was assessed by using the Japanese version of BADS. BADS includes six subtests as follows: the rule shift cards test, action program test, key search test, temporal judgment test, zoo map test, and the modified six elements test as shown in Table 1. We used standardized scores of the sum of all subtests. For the motor and executive assessment, score improvement was calculated as follows: a positive number indicates improvement, and a negative number indicates deterioration. Furthermore, we calculated percent improvement in the UPDRS part III score and standardized BADS score as follows: percent improvement = score improvement/baseline score.

\section{Statistical analysis}

Since the Kolmogorov-Smirnov test showed that change in the LED and percent improvement in UPDRS part III score in both the groups were non-normally distributed, we used the Spearman's correlation coefficients for the following analysis. Spearman's correlation coefficients of percent improvement in the BADS standardized score with simultaneous change in the LED and percent improvement in UPDRS part III were calculated. Patients' backgrounds, all of which were shown to be non-normally distributed by the Kolmogorov-Smirnov test, were compared between the two groups using Mann-Whitney $U$-test. Ratio of male to female was compared between the two groups using Fisher's exact test. Mean scores of motor and executive functions and 
LED were compared between baseline and the follow-up in each group using paired $t$-test. The level of significance was set at $p<0.05$ (two-tailed probability).

\section{Results}

Table 2 shows patients' backgrounds in both the groups. Ratio of male to female, age, and education showed no significant difference between the two groups. Table 3 shows change from baseline in mean motor and executive assessment scores and the LED of the prescribed drugs in both the groups. In the de novo group, motor function significantly improved with the dopaminergic medication. The mean standardized BADS score in both groups and the motor assessment score in the chronic medication group did not show significant changes.

In de novo group, levodopa/DDCI was prescribed to 15 patients, and six of the 15 patients took only levodopa/ DDCI. Dopamine agonists were prescribed to 9 patients, and all of the 9 patients took levodopa/DDCI and/or selegiline in combination; 13 patients took selegiline, and five of the 13 patients underwent selegiline monotherapy. In this group, percent improvement in the standardized BADS score showed a strong positive correlation with the LED $(\mathrm{r}=0.625, p<0.01)$ as shown in Figure 1. This significant correlation was preserved even excluding one outlier case with percent improvement of 1.667 ( $\mathrm{r}=0.594$, $p<0.01)$. Since the patients in both the groups had different backgrounds, such as age, education, disease duration, and motor and cognitive function at baseline, we performed a multiple regression analysis for these factors. The analysis using improvement in the standardized BADS score as a dependent variable showed only the LED significantly correlated with the executive improvement $(p<0.05)$ in the de novo group. However, percent improvement in the standardized BADS score $(\mathrm{r}=0.134, p=0.533$; shown in Figure 2) and the LED ( $\mathrm{r}=0.024, p=0.913)$ showed no significant correlation with percent improvement in UPDRS part III in the de novo group.

In the chronic medication group patients, percent improvement in the standardized BADS score showed significant negative correlation with the increase in the LED $(\mathrm{r}=-0.441, p<0.05$; shown in Figure 3 ). Multiple regression analysis for the same factors as performed in the de novo patients showed that improvement in the standardized BADS score did not correlate with any patient background factors including change in the LED. Percent improvement in standardized BADS score $(\mathrm{r}=0.381, p=0.088$; shown in Figure 4) and change in the LED ( $\mathrm{r}=-0.158, p=0.495)$ showed no significant correlation with percent improvement in UPDRS part III in the chronic medication group.

\section{Discussion}

In previous studies, executive function has been reported to be affected by dopaminergic medications in PD patients. ${ }^{5-8}$ However, the effect in clinical practice is inconsistent being positive or negative for executive function, and thus, dopaminergic drugs have not been used to treat cognitive dysfunction. Our study using the BADS scores, Spearman's correlation coefficients, and multiple regression analyses showed that improvement in executive function is positively correlated with the LED of the drugs prescribed to treat motor dysfunction in de novo PD patients without dementia. This confirms that dopamine affects executive function. However, improvement in the standardized BADS score did not correlate with simultaneous motor improvement in the de novo group patients. Previous studies showed that executive deficits in PD patients are linked to the frontostriatal circuit connecting the basal ganglia and frontal cortex. ${ }^{23,24}$ Dopamine regulates executive function in this circuit. ${ }^{25-27}$ On the other hand, dopaminergic medication is the gold standard for the treatment of motor symptoms. Therefore, these results in the de novo group suggest that motor and

Table 3 Change from baseline in mean motor and executive assessment scores and LED of prescribed drugs

\begin{tabular}{|c|c|c|c|c|}
\hline & \multicolumn{2}{|c|}{ De novo group } & \multicolumn{2}{|c|}{ Chronic medication group } \\
\hline & Baseline & $\begin{array}{l}\text { After the start of } \\
\text { the medication }\end{array}$ & Baseline & $\begin{array}{l}\text { After the adjustment } \\
\text { of the medication }\end{array}$ \\
\hline \multirow[t]{2}{*}{ UPDRS part III score } & $17.0 \pm 7.9$ & $10.0 \pm 5.6 * * *$ & $12.5 \pm 9.3$ & $9.6 \pm 6.6$ \\
\hline & $(5-36)$ & $(I-24)$ & $(2-45)$ & $(2 \mid-32)$ \\
\hline \multirow[t]{2}{*}{ Standardized BADS score } & $87.5 \pm 25.6$ & $91.0 \pm 25.7$ & $89.9 \pm 14.5$ & $86.3 \pm 19.0$ \\
\hline & $(12-124)$ & $(32-129)$ & $(61-104)$ & $(56-114)$ \\
\hline LED of prescribed & $0.0 \pm 0.0$ & $251.5 \pm 127.2^{* * *}$ & $425.7 \pm 3 \mid 4.8$ & $480.8 \pm 285.6$ \\
\hline dopaminergic drugs (mg/day) & & $(50-450)$ & $(75-1,445)$ & $(80-1,210)$ \\
\hline
\end{tabular}

Notes: $* * * p<0.001$ vs baseline. Data are presented as mean \pm SD.

Abbreviations: BADS, Behavioral Assessment of the Dysexecutive Syndrome; LED, levodopa equivalent dose; UPDRS, Unified Parkinson's Disease Rating Scale. 


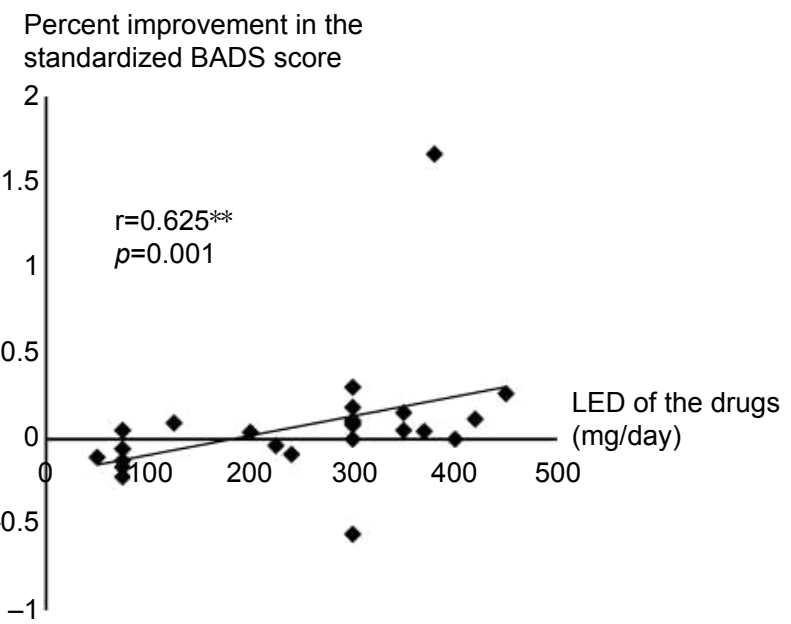

Figure I Distribution of percent improvement in the standardized BADS score and change in the LED in the de novo group.

Note: Percent improvement in the standardized BADS score showed a significant positive correlation with the LED $(* * p<0.01)$.

Abbreviations: BADS, Behavioral Assessment of the Dysexecutive Syndrome; LED, levodopa equivalent dose.

executive functions include a dopaminergic pathophysiology; however, the dopaminergic mechanisms of both functions are not identical.

The correlation between executive improvement and the LED was positive in the de novo group patients as shown in Figure 1. This positive correlation shows that executive function of patients who required larger LED to improve motor function tends to improve; However, that of patients with smaller LED tends to deteriorate. We consider this difference according to the LED using the "inverted U-shaped theory." Cools and D'Esposito

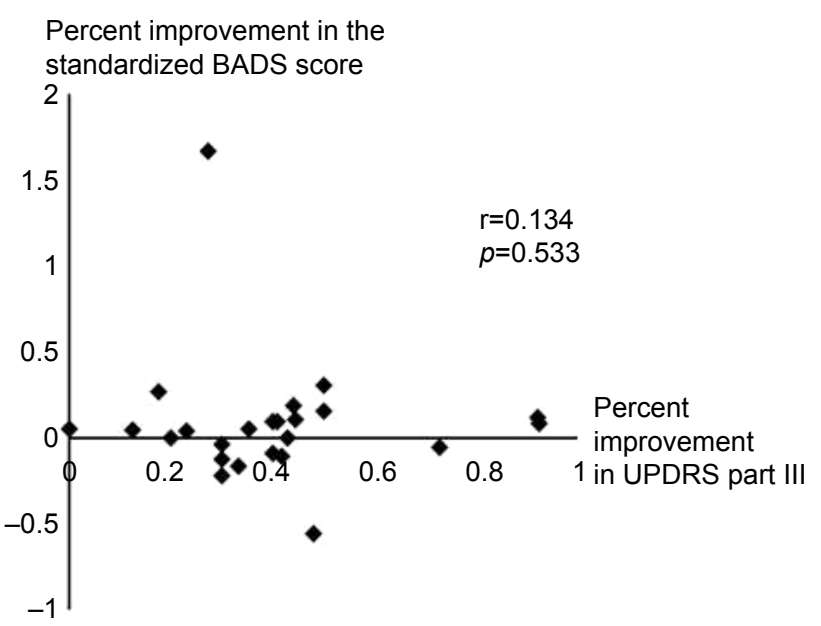

Figure 2 Distribution of percent improvement in the standardized BADS score and percent improvement in UPDRS part III in the de novo group.

Note: Percent improvement in the standardized BADS score did not correlate with the percent improvement in UPDRS part III.

Abbreviations: BADS, Behavioral Assessment of the Dysexecutive Syndrome; UPDRS, Unified Parkinson's Disease Rating Scale.

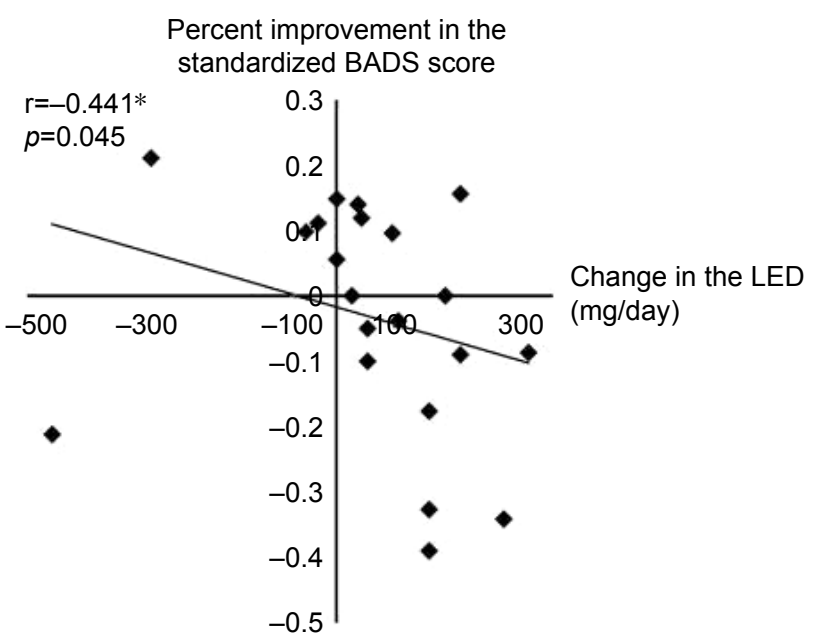

Figure 3 Distribution of percent improvement in the standardized BADS score and change in the LED in the chronic medication group.

Note: Percent improvement in the standardized BADS score showed a negative correlation with the change in the LED $(* p<0.05)$.

Abbreviations: BADS, Behavioral Assessment of the Dysexecutive Syndrome; LED, levodopa equivalent dose.

introduced this theory that indicates that too little and too much dopaminergic titers in the brain result in a decline in executive function, while an appropriate dopaminergic titer results in an improvement. ${ }^{8}$ Baseline dopaminergic titers of the de novo patients who required larger LED to improve motor function are speculated to be severely depleted at baseline, and the depleted dopaminergic titer supplemented with appropriate prescribed drugs resulted in appropriate dopaminergic titers and improved executive function. On the other hand, some compensatory mechanisms against

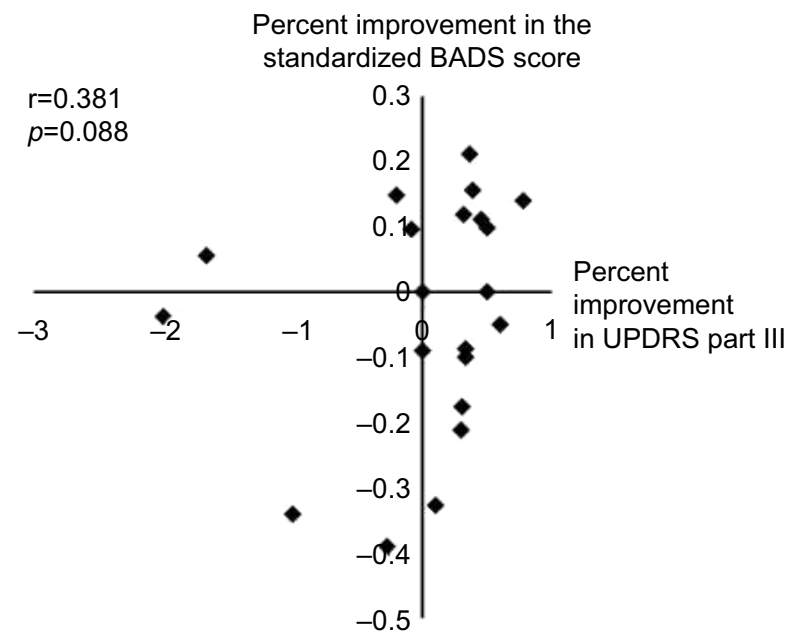

Figure 4 Distribution of percent improvement in the standardized BADS score and percent improvement in UPDRS part III in the chronic medication group.

Note: Percent improvement in the standardized BADS score showed no correlation with percent improvement in UPDRS part III.

Abbreviations: BADS, Behavioral Assessment of the Dysexecutive Syndrome; UPDRS, Unified Parkinson's Disease Rating Scale. 
dopaminergic neuron loss are shown in early PD patients and animal models. For example, dopamine release from residual striatal neuron in PD is elevated, and hyperactivity of residual neurons serves to maintain dopaminergic function. ${ }^{28}$ In early PD patients, dopamine receptors of postsynaptic neurons are upregulated. ${ }^{29}$ In the striatum of PD model mice, compensatory synthesis of dopamine by nondopaminergic neurons was observed. ${ }^{30}$ These compensatory mechanisms have a possibility to have contributed to the effect of dopaminergic medication in the de novo patients who required smaller LED to improve motor function as follows. Such patients are speculated to have larger endogenous dopamine and/or grater compensatory mechanisms, and their baseline dopaminergic titers are speculated to be relatively strong. Therefore, additional dopaminergic drugs made total dopaminergic titer too much and deteriorated executive function in such patients.

For the chronic medication group patients already taking dopaminergic drugs, there was a negative correlation between change in the LED and executive improvement. According to the "inverted U-shaped theory," the results of these patients signify that additional medication increases the total dopaminergic titer too much and causes a decline in executive function. However, multiple regression analysis showed that improvement in executive function did not directly correlate with change in the LED in the chronic medication group in contrast to the de novo patients. Therefore, other factors are suggested to contribute to the change in executive function with the additional LED. For example, executive impairment in PD is due to a deficit not only in dopamine, but also in other neurotransmitters. In PD patients with dementia, donepezil, a cholinesterase inhibitor, improved executive function. ${ }^{31}$ In PD patients with no dementia, executive function improved with atomoxetine, a selective norepinephrine reuptake inhibitor. ${ }^{32}$ Based on these reports, not only dopamine, but also acetylcholine and norepinephrine modulate executive function. These neurotransmitters except dopamine may have contributed to the effects of the medication, but the real contributing factor is uncertain. Therefore, the effect of dopaminergic drugs on the executive function in the chronic dopaminergic medication group was inconsistent, and dopaminergic dependency of executive function is weaker in the chronic medication stage than in the de novo stage.

The medication administered to our patients was single use or combinations of levodopa/carbidopa, levodopa/ benserazide, and dopamine agonists and selegiline. The affinity for the dopamine receptor subtypes differs in these drugs.
In particular, the dopamine D1 receptor affinity varies greatly depending on the medication. For example, ropinirole and pramipexole bind poorly to the D1 receptor. In contrast, the dopamine D2 receptor affinity does not differ so much according to these drugs. ${ }^{33}$ A correlation between change in executive function and change in the LED in the de novo group was common to all medication types. Therefore, the change in both executive and motor functions seen in our present study may be mediated for the most part by D2 receptor effects. Actually the dopamine D2 receptor is the primary and common target for the anti-Parkinsonian action of dopamine agonists, because there is a correlation between the therapeutic concentrations of dopamine agonists and their D2 receptor affinities. ${ }^{34}$ Executive function is regulated by D2 receptor function. ${ }^{35-37}$

We did not consider the body weight of the patients, because serum and cerebrospinal fluid concentrations and bioavailability of prescribed drugs differ greatly from patient to patient. Even if the body weight is calculated, the dose of given drugs per unit weight is not useful. Our results show that the executive function of drug-naïve patients improves dose-dependently with dopaminergic medications in clinical practice.

\section{Conclusion}

In de novo PD patients, improvement in executive function with dopaminergic medication has a direct positive correlation with the LED of the prescribed drugs to treat motor dysfunction. After the chronic dopaminergic treatment, correlation between improvement in executive function and additional dopaminergic medications becomes negative. However, this negative correlation is not a direct correlation. Therefore, the effect of dopaminergic medication on executive function may get relatively weak during the chronic dopaminergic medication.

Improvement in executive function with initiation/ adjustment of dopaminergic drugs did not correlate with the simultaneous improvement in the motor function. Executive function includes dopaminergic mechanisms, but it is not identical with the dopaminergic mechanism of motor function.

\section{Acknowledgments}

This study was partly supported by scholarship donation from Novartis Pharma KK, Mitsubishi Tanabe Pharma Corporation, Bayer Yakuhin, Ltd., Japan Blood Products Organization, Daiichi Sankyo Co., Ltd., Bristol-Myers Squibb Company, and FP Pharmaceutical Corporation. 
The abstract of this paper was presented at the 21st International Congress of Parkinson's Disease and Movement Disorders as a poster presentation with interim findings. The poster's abstract was published in "Poster Abstracts" in Movement Disorders, Volume 32, Issue Supplement S2, June 2017: DOI: 10.1002/mds.27087.

\section{Disclosure}

The authors report no conflicts of interest in this work.

\section{References}

1. Caviness JN, Driver-Dunckley E, Connor DJ, et al. Defining mild cognitive impairment in Parkinson's disease. Mov Disord. 2007; 22(9):1272-1277.

2. Goldman JG, Weis H, Stebbins G, Bernard B, Goetz CG. Clinical differences among mild cognitive impairment subtypes in Parkinson's disease. Mov Disord. 2012;27(9):1129-1136.

3. Pellecchia MT, Picillo M, Santangelo G, et al. Cognitive performances and DAT imaging in early Parkinson's disease with mild cognitive impairment: a preliminary study. Acta Neurol Scand. 2015; 131(5):275-281.

4. Siepel FJ, Brønnick KS, Booij J, et al. Cognitive executive impairment and dopaminergic deficits in de novo Parkinson's disease. Mov Disord. 2014;29(14):1802-1808.

5. Pillon B, Czernecki V, Dubois B. Dopamine and cognitive function. Curr Opin Neurol. 2003;16(Suppl 2):S17-S22.

6. Lewis SJ, Slabosz A, Robbins TW, Barker RA, Owen AM. Dopaminergic basis for deficits in working memory but not attentional set-shifting in Parkinson's disease. Neuropsychologia. 2005;43(6):823-832.

7. Lange KW, Paul GM, Robbins TW, Marsden CD. L-dopa and frontal cognitive function in Parkinson's disease. Adv Neurol. 1993; 60:475-478.

8. Cools R, D'Esposito M. Inverted-U-shaped dopamine actions on human working memory and cognitive control. Biol Psychiatry. 2011; 69(12): e113-e125.

9. Takahashi H, Kato M, Takano H, et al. Differential contributions of prefrontal and hippocampal dopamine $\mathrm{D}(1)$ and $\mathrm{D}(2)$ receptors in human cognitive functions. J Neurosci. 2008;28(46):12032-12038.

10. Fallon SJ, Smulders K, Esselink RA, van de Warrenburg BP, Bloem BR, Cools R. Differential optimal dopamine levels for set-shifting and working memory in Parkinson's disease. Neuropsychologia. 2015; $77: 42-51$.

11. Green J, McDonald WM, Vitek JL, et al. Cognitive impairments in advanced PD without dementia. Neurology. 2002;59(9):1320-1324.

12. Riggeal BD, Crucian GP, Seignourel P, et al. Cognitive decline tracks motor progression and not disease duration in Parkinson patients. Neuropsychiatr Dis Treat. 2007;3(6):955-958.

13. Amboni M, Cozzolino A, Longo K, Picillo M, Barone P. Freezing of gait and executive functions in patients with Parkinson's disease. Mov Disord. 2008;23(3):395-400.

14. Nocera JR, Price C, Fernandez HH, et al. Tests of dorsolateral frontal function correlate with objective tests of postural stability in early to moderate stage Parkinson's disease. Parkinsonism Relat Disord. 2010;16(9):590-594.

15. Norris G, Tate RL. The behavioral assessment of the dysexecutive syndrome (BADS): ecological, concurrent and construct validity. Neuropsychol Rehabil. 2000;10(1):33-45.

16. Wilson BA, Evans JJ, Emslie H, Alderman N, Burgess P. The development of an ecologically valid test for assessing patients with a dysexecutive syndrome. Neuropsychol Rehabil. 1998;8(3):213-228.

17. Evans JJ, Chua SE, McKenna PJ, Wilson BA. Assessment of the dysexecutive syndrome in schizophrenia. Psychol Med. 1997;27(3): 635-646.
18. Kamei S, Hara M, Serizawa K, et al. Executive dysfunction using behavioral assessment of the dysexecutive syndrome in Parkinson's disease. Mov Disord. 2008;23(4):566-573.

19. Perfetti B, Varanese S, Mercuri P, Mancino E, Saggino A, Onofrj M. Behavioural assessment of dysexecutive syndrome in Parkinson's disease without dementia: a comparison with other clinical executive tasks. Parkinsonism Relat Disord. 2010;16(1):46-50.

20. Teramoto H, Morita A, Ninomiya S, Shiota H, Kamei S. Relation between freezing of gait and frontal function in Parkinson's disease. Parkinsonism Relat Disord. 2014;20(10):1046-1049.

21. Gibb WR, Lees AJ. The relevance of the Lewy body to the pathogenesis of idiopathic Parkinson's disease. J Neurol Neurosurg Psychiatry. 1988;51(6):745-752.

22. Tomlinson CL, Stowe R, Patel S, Rick C, Gray R, Clarke CE. Systematic review of levodopa dose equivalency reporting in Parkinson's disease. Mov Disord. 2010;25(15):2649-2653.

23. Dubois B, Pillon B. Cognitive deficits in Parkinson's disease. J Neurol. 1997;244(1):2-8.

24. Owen AM. Cognitive dysfunction in Parkinson's disease: the role of frontostriatal circuitry. Neuroscientist. 2004;10(6):525-537.

25. Nieoullon A. Dopamine and the regulation of cognition and attention. Prog Neurobiol. 2002;67(1):53-83.

26. Sawamoto N, Piccini P, Hotton G, Pavese N, Thielemans K, Brooks DJ. Cognitive deficits and striato-frontal dopamine release in Parkinson's disease. Brain. 2008;131(Pt5):1294-1302.

27. Jokinen P, Karrasch M, Brück A, Johansson J, Bergman J, Rinne JO. Cognitive slowing in Parkinson's disease is related to frontostriatal dopaminergic dysfunction. J Neurol Sci. 2013;329(1-2):23-28.

28. Zigmond MJ, Abercrombie ED, Berger TW, Grace AA, Stricker EM. Compensations after lesions of central dopaminergic neurons: some clinical and basic implications. Trends Neurosci. 1990;13(7):290-296.

29. Hägglund J, Aquilonius SM, Eckernäs SA, et al. Dopamine receptor properties in Parkinson's disease and Huntington's chorea evaluated by positron emission tomography using 11C-N-methyl-spiperone. Acta Neurol Scand. 1987;75(2):87-94.

30. Kozina EA, Kim AR, Kurina AY, Ugrumov MV. Cooperative synthesis of dopamine by non-dopaminergic neurons as a compensatory mechanism in the striatum of mice with MPTP-induced Parkinsonism. Neurobiol Dis. 2017;98:108-121.

31. Dubois B, Tolosa E, Katzenschlager R, et al. Donepezil in Parkinson's disease dementia: a randomized, double-blind efficacy and safety study. Mov Disord. 2012;27(10):1230-1238.

32. Marsh L, Biglan K, Gerstenhaber M, Williams JR. Atomoxetine for the treatment of executive dysfunction in Parkinson's disease: a pilot open-label study. Mov Disord. 2009;24(2):277-282.

33. Wood M, Dubois V, Scheller D, Gillard M. Rotigotine is a potent agonist at dopamine $\mathrm{D} 1$ receptors as well as at dopamine $\mathrm{D} 2$ and $\mathrm{D} 3$ receptors. Br J Pharmacol. 2015;172(4):1124-1135.

34. Seeman P. Antiparkinson therapeutic potencies correlate with their affinities at dopamine D2(High) receptors. Synapse. 2007;61(12): 1013-1018.

35. Volkow ND, Gur RC, Wang GJ, et al. Association between decline in brain dopamine activity with age and cognitive and motor impairment in healthy individuals. Am J Psychiatry. 1998;155(3):344-349.

36. Ballard ME, Dean AC, Mandelkern MA, London ED. Striatal dopamine D2/D3 receptor availability is associated with executive function in healthy controls but not methamphetamine users. PLoS One. 2015; 10(12): 0143510.

37. Chen PS, Yang YK, Lee YS, et al. Correlation between different memory systems and striatal dopamine D2/D3 receptor density: a single photon emission computed tomography study. Psychol Med. 2005; 35(2):197-204. 


\section{Publish your work in this journal}

Neuropsychiatric Disease and Treatment is an international, peerreviewed journal of clinical therapeutics and pharmacology focusing on concise rapid reporting of clinical or pre-clinical studies on a range of neuropsychiatric and neurological disorders. This journal is indexed on PubMed Central, the 'PsycINFO' database and CAS,

and is the official journal of The International Neuropsychiatric Association (INA). The manuscript management system is completely online and includes a very quick and fair peer-review system, which is all easy to use. Visit http://www.dovepress.com/testimonials.php to read real quotes from published authors.

Submit your manuscript here: http://www.dovepress.com/neuropsychiatric-disease-and-treatment-journal 